\title{
Geometrical Assessment of Ocular Exposure to Envirmmental UV Radiation -Implications for Ophthalmic Epidemiology
}

\author{
David H. Sliney
}

\begin{abstract}
Epidemiological studies of the influence of environmental ultraviolet radiation (UVR) in the development of cataract, pterygium, droplet keratopathies and age-related macular degeneration have produced inconsistent findings. The lack of consistent results may be due largely to either incomplete or erroneous estimates of outdoor UV exposure dose. Geometrical factors dominate the determination of UVR exposure of the eye. The degree of lid opening limits ocular exposure to only those rays entering at angles near the horizon. Clouds redistribute overhead UVR to the horizon sky. Mountains, trees and building shield the eye from direct sky exposure. Most ground surfaces reflect little UVR. The result is that the highest UVR exposure occurs during light overcast where the horizon is visible and ground surface reflection is high. By contrast, exposure in a high mountain valley with green foliage results in a much lower ocular dose. Other findings of these studies show that retinal exposure to light and UVR in daylight occurs largely in the superior retina. J Epidemiol, $1999 ; 9: \mathrm{S} 22-\mathrm{S} 32$.
\end{abstract}

light, ultraviolet radiation, ocular exposure, cataract, pterygium

The earlier age of onset of cataract in equatorial zones has been a subject of interest for decades, and has led to a number of theories to explain this latitudinal dependence. Although some studies have concluded (and it has long been argued) that exposure of the human eye to UVR plays an etiologic role in the development of ocular diseases, including cataract ${ }^{187}$, this role in cataractogenesis has been questioned by others ${ }^{9-12}$. Although photokeratitis is unquestionably caused by UVR reflection from the snow ${ }^{12-19)}$, pterygium and droplet keratopathies are less clearly related to UVR exposure ${ }^{12,20)}$. Even more under debate are theories that suggest that UVR and intense light may affect retinal disease 2, 12, 13, 21-23). To answer these questions, far better ocular dosimetry is required. Epidemiologic studies can arrive at erroneous conclusions if assignments of exposure are seriously in error, and assumptions regarding relative exposures have been argued to be incorrect ${ }^{8,13)}$.

Before one can improve on current epidemiological studies of ocular deceases (or determine the most effective UVR protective measures), it is necessary to characterize the primary sources of UVR exposure to the eye. For more than two decades, my laboratory at the US Army Center for Health
Promotion and Preventive Medicine (USACHPPM) has been studying the geometrical exposure factors by measuring UV skylight distributions under different environmental conditions and the impact of different protective measures. Studies were also conducted to determine how outdoor scene luminance (brightness) affects the degree of lid opening in outdoor subjects, and how eyewear affects UVR exposure.

\section{WHAT TO MEASURE?}

Our program of environmental UVR measurements was initiated with the goal of how to improve protective measures and to better quantify the UVR exposure of the skin and eye. The first question to be answered was: What part of the terrestrial solar spectrum should be measured? And, what spectral instrument response would mimic any known action spectrum for the adverse effects to be considered? Finally: What direction(s) do we measure with what geometrical field-of-view (FOV) would be relevant to human ocular exposure conditions outdoors. Figure 1 shows the different components of ocular exposure which must be considered. Answering these questions is also critically important in improving epidemiological

Address for correspondence : David H. Sliney, Ph.D., Laser/Optical Radiation Program, U.S. Army Center for Health Promotion and Preventive Medicine, Aberdeen Proving Ground, Maryland, USA. 


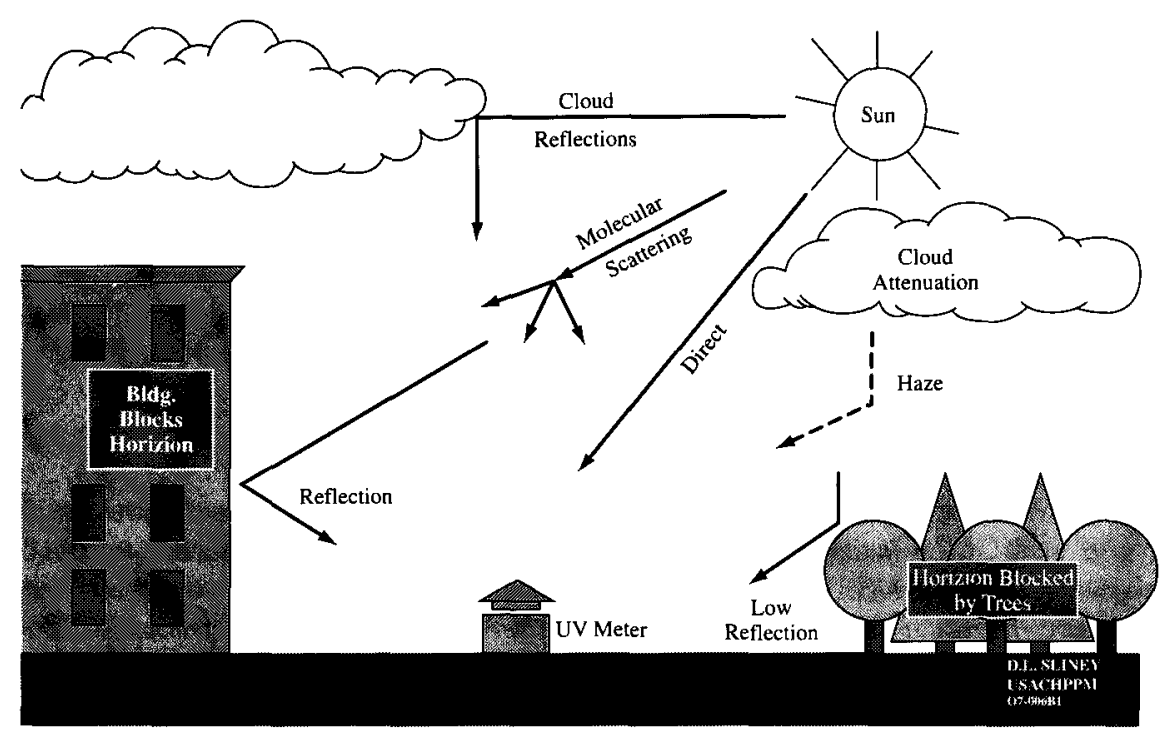

Figure 1. More than 50-percent of ambient UVR arrives from skylight scatter and cloud reflection and scatter. One can actually receive a"sunburn" whilst in the shade, if sufficient blue sky is visible. Of ground covers, only snow is highly reflective.

studies. It is therefore fitting to examine what spectral sensitivity and threshold information is known about the different ocular risks.

\section{Lenticular Opacities}

As noted previously, the evidence that exposure of the human eye to UVR plays an etiologic role in the development of cataract has continued to evoke some disagreement ${ }^{1-12)}$. Ultraviolet cataract can be produced experimentally in animals from exposures to UVR of wavelengths between $295 \mathrm{~nm}$ and $325 \mathrm{~nm}$ (and perhaps to $400 \mathrm{~nm}$ ). Action spectra (revealing the relative hazard of each wavelength) can only be obtained from the laboratory studies, and these have shown that anterior cortical and posterior subcapsular cataract can be produced by intense exposure delivered over a period of days ${ }^{35}$ ).

At wavelengths less than $290 \mathrm{~nm}$ all incident UVR is absorbed in the cornea, and as the wavelength increases to 300 $\mathrm{nm}$, trace amounts of UVR reach the lens, and by $315 \mathrm{~nm}$, the cornea transmits nearly half the incident radiation. UVR is absorbed largely in the lens at wavelengths greater than approximately $340 \mathrm{~nm}^{2-4}$. Human cortical cataract has been linked to chronic, life-long UV-B radiation exposure ${ }^{6)}$ and most animal studies ${ }^{3-5)}$ and suggest that acute cataracts result primarily from UV-B radiation, however, some in vitro biochemical studies suggest that UV-A radiation may also contribute to accelerated ageing of the lens ${ }^{24-25)}$. It should be noted that the wavelengths between 295 and $325 \mathrm{~nm}$ are also in the wavelength region that increases significantly with ozone depletion. Since there is an earlier age of onset of cataract in equatorial zones, UVR exposure has frequently been one of the most appealing of a number of theories to explain this latitudinal dependence.

\section{Ophthalmoheliosis--The Coroneo Effect}

For any greatly delayed health effect, such as cataract, pterygium, or retinal degeneration, it is critical to determine the actual dose distribution at critical locations. A factor of great practical importance is the actual UVR which reaches the germinative layers of any tissue structure. In the case of the lens, the germinative layer where lens fiber cell nuclei are located is of great importance. The DNA in these cells is normally well shielded by the parasol effect of the irides. Since the shortest and most direct pathway of UVR to the inferior germinative area of the lens is from the extreme temporal direction, it has been speculated that side exposure is particularly hazardous ${ }^{26-28)}$. Coroneo ${ }^{27)}$ has suggested that focussing of very peripheral rays by the temporal edge of the cornea--those which do not even reach the retina, can enter the pupil and reach the equatorial region as shown in Figure 2. He termed this effect which can produce a concentration of UVR at the nasal side of the limbus and lens, "ophthalmoheliosis." He argues the more frequent onset of cataract in the nasal quadrant of the lens and the formation of pterygium (described below) in the nasal region of the cornea ${ }^{26)}$. Figure 3 shows the percentage of cortical cataract which actually first appears in each quadrant of the lens from the data of Klein, et al. in their study of a population in the mid-west of the US, in Beaver Dam, Wisconsin ${ }^{11)}$. This relationship is highly consistent with the Coroneo hypothesis. 


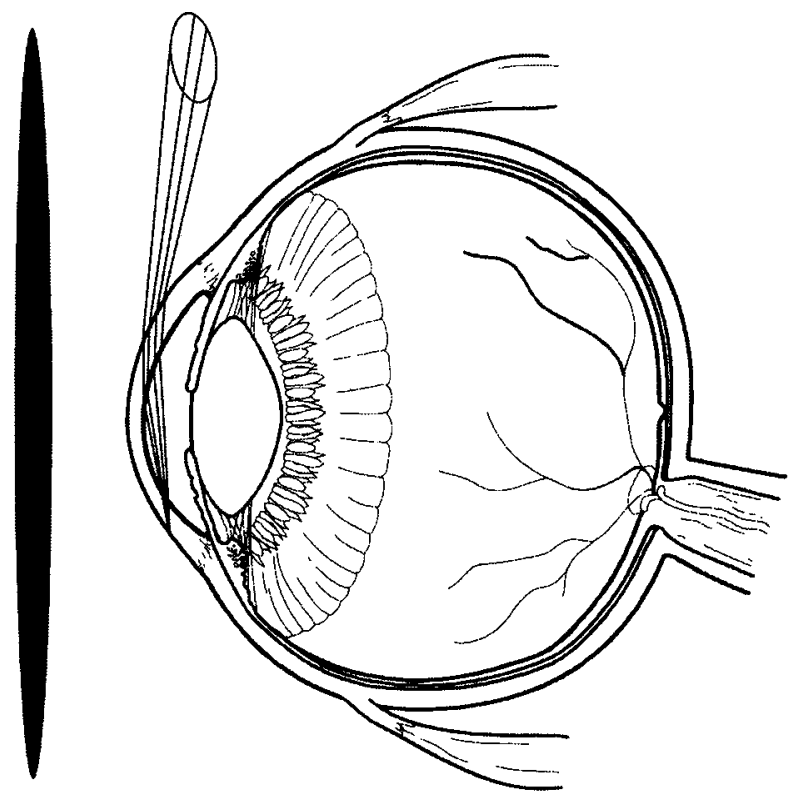

Figure 2. The Coroneo Effect. Oblique optical rays from the extreme temporal edge of the cornea can be refracted to pass through the pupil and arrive at the germinative layer of the lens (the equatorial zone). The path length through the absorbing lens is approximately the same as a ray directly through the lens along the optical axis; hence approximately $1 \%$ of this oblique, 300 $\mathrm{nm}$ radiation will reach the equator [24]. A typical sunglass lens does not block these rays.

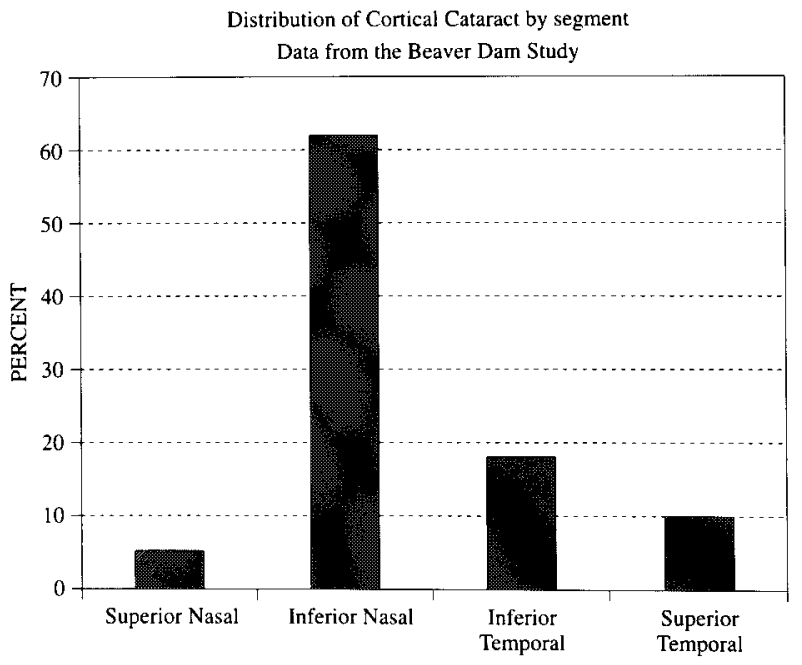

AmJPubHth, 82(12):1658-1662(1992)

Figure 3. Distribution of cortical cataract found in the Beaver Dam Study'? There appear to be a five-fold greater likelihood of finding opacities in the inferior nasal quadrant. There was little difference whether or not the estimated exposure was more than or less than the author's estimate of outdoor exposure to sunlight in "Wisconsin Sun Years". 


\section{Pterygium and Droplet Keratopathies}

Although the acute UVR effects of photokeratitis and photoconjunctivitis clearly result from UV-B reflection from the snow ${ }^{12-19)}$, it is still not clear whether UVR is the principal etiologic factor in pterygium and droplet keratopathies, and how much wind and dust may play a role ${ }^{20)}$. Pterygium, a fatty growth over the cornea, is common in central Australian and ocean-island residents (where both UVR and wind exposure are prevalent), UVR ${ }^{12,20,2 \pi}$, and here, the Coroneo Effect may well play a role ${ }^{2 m}$.

\section{Cancers}

Cancers arising from chronic exposure to UVR, particularly from UV-B (280-315 nm), have been demonstrated for the skin $^{4,25,29-31)}$; however little is known about the potential contribution of UVR to ocular cancers and ocular melanoma. It is interesting that whilst corneal cancers do occur in cattle, they are almost unknown in humans ${ }^{32-33}$. Retinal melanomas are not known to be associated preferentially with the most illuminated retinal zones.

\section{Erythema}

Ultraviolet erythema ("sunburn" or reddening of the skin -$200 \mathrm{~nm}$ to $400 \mathrm{~nm}$ ) applies to the lids of the eye. This effect appears several hours after an acute exposure and generally lasts from 8 hours to 72 hours depending upon degree exposure and spectral region ${ }^{34)}$. The ACGIH and ICNIRP guideline for UVR also protects against erythema with an added safety factor for all skin types. The action spectra ${ }^{34-35)}$ there- fore can be compared to that of the ACGIH/ICNIRP $S(\lambda)$ as shown in Figure 4.

\section{Retinal Effects}

Theories which suggest that UVR (and even light) may contribute to some age-related retinal diseases are very much in debate $^{1,2)}$. Acute exposure to light can produce a photochemical injury to the retina (principally from visible $400 \mathrm{~nm}$ to 550 $\mathrm{nm}$ blue light with generally only a small UV contribution). This often is referred to as "blue light" photoretinitis, e.g., solar retinitis which may lead to a permanent scotoma ${ }^{1,21,37)}$. Prior to conclusive animal experiments two decades ago, solar retinitis was thought to be a thermal injury mechanism ${ }^{23}$. Unlike thermal retinal injury, there is no image-size dependence. The ICNIRP/ACGIH B $(\lambda)$ weighted integrated radiance limit $\mathrm{L}_{B}$ is $100 \mathrm{~J} /\left(\mathrm{cm}^{2} \mathrm{sr}\right)$ averaged over a right circular cone of 0.011 radian for durations up to $10^{4}$ seconds $(2.8$ hours); although a larger averaging angle is generally used for durations of the order of $1000 \mathrm{~s}$ or greater where a non-fixation visual task is involved. Normally the crystalline lens absorbs 99\% or more of the UVR which could enter the eye. The B ( $\lambda$ ) function has a value of 0.01 from $310-380 \mathrm{~nm}{ }^{50)}$; hence, the hazardous integrated radiance for a pure UV-A source is about $10 \mathrm{~kJ} /\left(\mathrm{cm}^{2} \mathrm{sr}\right)$. However, in infancy or during cataract surgery after the cataractous lens has been removed (aphakia) and before a UV absorbing intraocular lens implant has been placed in the eye (pseudophakia), the retina is quite vulnerable. The aphakic hazard spectrum $A(\lambda)$ can be applied for a hazard analysis.

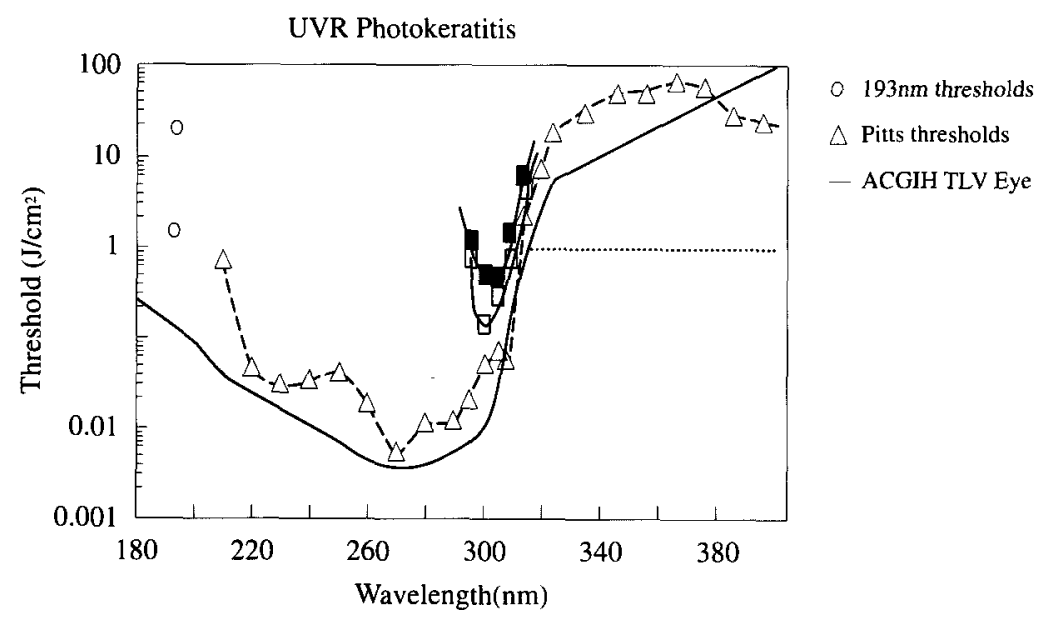

Figure 4. The reciprocul, normalized value is the ICNIRP UV Hazard Function $S(\lambda)$. The solid line describes approximately the relative spectral risk for photokeratitis. Actual thresholds for photokeratitis in experimental animals are also provided as triangles, and for acute cataract as squares. Open squares were reversible thresholds; solid squares for permanent opacities in animal studies of Pitts et al. 


\section{The Choice of Spectral Response}

Although we have measured the changing spectral distribution of sunlight at different solar zenith angles, the only feasible means to measure the geometrical variations of biologically weighted sunlight is to employ broad-band monitors. After a thorough review of the known biological action spectra, we chose to use stationary and field-portable instruments which followed the internationally accepted action spectra for UV safety, since it followed the DNA damage action spectrum and the action spectrum for acute corneal injury, photokeratitis. The public health guidelines of the International Commission for Non-Ionizing Radiation Protection ${ }^{13,50)}$ guideline is an $S(\lambda)$ weighted daily (8-hour) exposure $\mathrm{H}_{\text {eff }}$ of $3 \mathrm{~mJ} / \mathrm{cm}^{2}$ or 30 $\mathrm{J} / \mathrm{m}^{2}$, which is just below the level which produces a barely detectable increase in corneal light scatter.

\section{AMBIENT UVR MEASUREMENTS}

The extensive UVR measurements of sky conditions, ground surfaces, mannequin exposure, etc. are too complex to detail here. Only a few broad observations and an example can be provided. We concluded from our studies that serious errors result if one attempts to estimate UVR ocular exposure by relying on ones general experience of the meteorological environment and assume that the geometrical distribution of UVR is similar to what we can see in the visible spectrum. The aim of our UVR measurement program and ocular dosimetry studies has been to gain a far more complete picture and quantitative description of the ambient UVR environment when persons are outdoors.
By contrast with visible light, UVR is very strongly scattered, and the scatter increases greatly with decreasing wavelength. For this reason (known as "Rayleigh Scattering"), the sky is blue. If one could see only in the UV-B spectrum (280$315 \mathrm{~nm}$ ), a clear sunny sky would appear to be a sun barely visible through a very heavy fog or haze; ground shadows would be very fuzzy; green grass (which has a reflectance of only $1 \%$ ) would appear pitch black; sand and most ground surfaces would appear very dark grey, like an asphalt roadway; and most UV-B exposure arriving at the face would be from diffuse scatter and not from direct sunlight. The eye's exposure would be totally dominated by ground reflection. Standing over water, one would see a reflection of the grey sky, and ocular exposure to UVR would be increased. Cumulus clouds would appear darker or lighter than the brilliant "blue" portion of the sky, depending upon direct sunlight exposure. Figure 5 illustrates that the entire field of view reflects the sky's UVR.

\section{Environmental UVR Measurements}

Our group has also measured the geometrical distribution of environmental UVR. These measurements clearly show that the greater portion of UVR reaches the eye from scatter from clouds and reflections from ground surfaces than from direct sunlight $7,8,13,28$. Therefore, directional UVR measurements of limited fields are more relevant in determining the exposure to the eye than the typically reported "global" measurements which describe the spectral irradiance or erythemally effective irradiance falling upon a horizontal "cosine-corrected" diffuser. We also sought to examine those environmental conditions which would cause a high ratio of ultraviolet radiation to out-

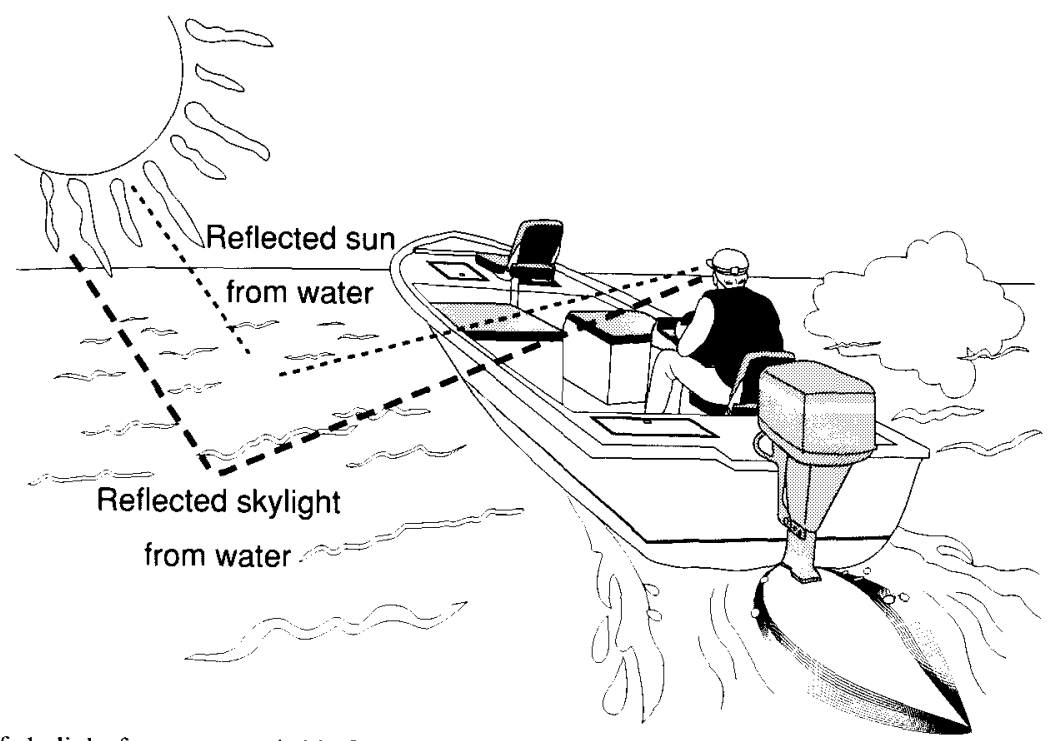
Figure 5. Reflections of skylight from water. Aside from snow, open water reflects the greatest amount of UVR into the eye. Not only
direct sunlight, but the entire sky's UVR is reflected. 
door luminance. With this information and the characterization of the dependance of the vertical visual field upon luminance, a relationship between the environment and the total UVR exposure to the eye can be found.

\section{Ocular Exposure and the Field Of View}

Because our earlier studies showed that UVR exposure is not very directional and our lids greatly limit ocular UVR exposure, we had to devise an approach different from that normally employed when measuring sunlight. We approached the problem by deriving a formula that split up the two major components of ocular radiant exposure $\mathrm{H}$ : the ground reflection component $\mathrm{H}_{g}$ and the sky radiance source component $\mathrm{H}_{s}$. Each of these components includes a physically determined radiance and a geometrical factor, the solid angle of acceptance determined by lid opening.

$$
\mathrm{H}=\mathrm{H}_{\text {sky }}+\mathrm{H}_{\text {gound }}=\mathrm{H}_{\mathrm{s}}+\mathrm{H}_{\mathrm{g}}
$$

Each component is determined by the brightness of the scene viewed by the eye. This brightness, or radiance $\mathrm{L}$, and the eye's solid angle of acceptance (i.e., field-of-view) in units of steradians (sr) for that portion of each hemisphere in one's total visual field. This approach may sound at first to be an overly complicated way of determining total exposure; however, since the UVR source is our total surrounding and not just a single very small source as the sun by itself, this procedure is essential to avoid substantial errors.

Radiance has units of watts-per-square-centimeter-per-steradian. To better understand the concept of radiance, consider the following example: If you stand in a dark room with one window, the amount of light falling upon your face is quite high when standing at the window, but as you back away, the facial illumination drops greatly. Although the total light (or UVR for that matter) entering the eye and passing through the lens changes greatly, the luminance (brightness) of the outdoor scene does not change. In photography, one actually measures the visible radiance (known as luminance) with a spot meter when determining the proper film exposure. Indeed, it is convenient to calculate the light level falling on one's face by multiplying the luminance or radiance (brightness of the source) by the solid angle of the source (the window) to obtain the illumination or irradiance. This method has the great advantage that one need merely determine the source radiance (e.g., the sky) and then determine the solid angle corresponding to a lid opening, or the field-of-view determined by a headwrap or hat. This is a somewhat similar approach to that used by Rosenthal, and termed the Optical Ambient Exposure Ratio (OAER) ${ }^{10)}$. The additional factors that we have added are the impact of the greatly reduced solid-angle of acceptance created by squinting and the importance of horizon sky radiance, and whether the horizon sky can be observed. Neither of these new factors affect the outcome of the Chesapeake Bay Waterman's Study, but we feel that an increased risk factor may result from considering this approach for future epidemiological studies.

\section{Measuring the Angle of Acceptance for Ocular Exposure}

Facial features, such as the eyelids, brow ridge, and cheek, function to limit the "aperture" for light and UVR reaching the cornea and lens. While bone structure and other facial characteristics are genetically determined, some attributes such as the degree of eyelid opening vary with environmental lighting conditions. Our studies showed that of theses factors, eyelid opening is dominant in limiting UVR exposure of the anterior structures of the eye. The eyelids react to the visible brightness, or luminance, of the scene being viewed, attenuate the amount of light entering the eye and affects the regional area of the retina being illuminated, but do not reduce the local concentration of light at the retina (the retinal irradiance) in the illuminated area. Because the UV-B arriving at the earth's surface is so strongly scattered, much of the UVR incident on the eye comes from skylight near the horizon. Hence, the lid opening is critically important in determining UV-B exposure of the cornea and lens ${ }^{8}$. A correlation between the luminance and the amount of lid closure is needed to determine the amount of UVR which reaches the eye in different conditions.

In pilot studies, it was clear that the upper lid's position varied with scene luminance over a wide range of luminances, but the lower lid did not begin to raise except in the very brightest conditions; hence, only the upper lid position was measured. Initially, we tried to measure lid opening photographically, but found this too difficult and unreliable. Since the upper lid position acts as a "shade" and limits the vertical visual field-ofview (FOV), we designed a simple procedure to measure only the upper limit of the FOV. This permitted us to calculate the upper acceptance angle of light into the eye.

\section{Techniques Used in Measuring Lid Opening}

A total of fifty adult subjects took part in this study. Each subject stood at least five minutes outdoors to fully adapt to sunlight prior to measurements of lid opening. To measure the upper FOV angle, an object was lowered from a tall surveyor's pole into each subject's visual field. Each subject stood a fixed distance from the pole and was asked to fixate at a horizontal point on the pole. The angle at which the object was first detected was considered to be the maximum vertical acceptance angle of light reaching the eye. Figure 6 sumarizes the FOV measurement $s$.

Since the goal of this series of studies was to develop an algorithm for the amount of UVR exposure an average person receives while outside during a lifetime, an attempt was made to model human behavior as accurately as possible. While walking outdoors, most people do not look straight ahead, but down at an angle which averages to approximately 15 degrees 


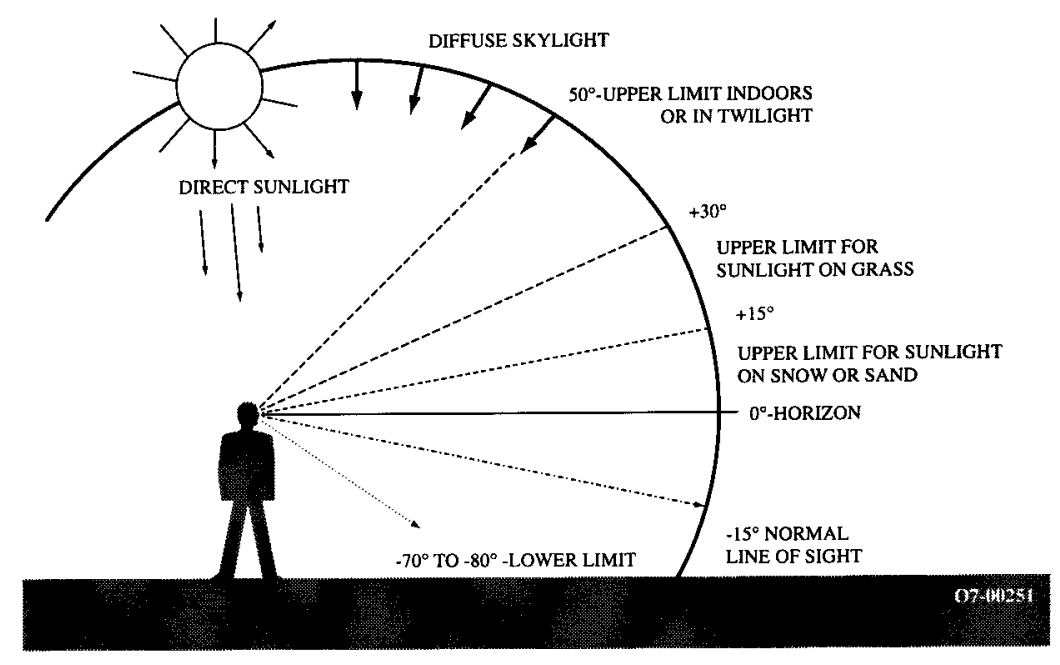

Figure 6. Field of view values for subjects showing the spread of values for individuals.

below the horizontal ${ }^{13,38}$. To account for this estimate, the subjects were instructed to look at an object at 15 degrees below the horizontal. With this line-of-sight fixed, the upper vertical acceptance angle was measured. In addition, a second FOV measurement was taken while the subjects looked horizontally. The majority of tests show that the acceptance angle for each subject as measured from the line-of-sight remained the same regardless of this viewing angle. However, the acceptance angle often increased as much as 10-15 degrees when the lineof-sight was below the horizontal. This might have been expected since the luminance of the ground is usually lower than that of the sky, causing less squinting. A variety of different luminance conditions (measured with a hand-held Minolta Luminance Meter) were studied, ranging from a white wall on a bright sunny afternoon to dense trees on a heavily overcast morning. This luminance range spanned three orders of magnitude, reaching as low as $170 \mathrm{~cd} / \mathrm{m}^{2}$, and as high as $15,000 \mathrm{~cd} / \mathrm{m}^{2}$.

To assure that the luminance was the primary determinant in the amount of squinting, subjects were also tested wearing two different pairs of sunglasses. Both pair reduced the luminance roughly by a factor of four (3.8 and 4.3), although one had lenses with an orange tint that blocked more strongly in the blue part of the spectrum while the other was a neutral tone lens. Since the FOV for no sunglasses was also measured at this time, there was an opportunity to compare results with previous measurements to determine the precision of the technique. Under the same luminance conditions, the upper FOV angle typically spanned a range of about 25 degrees. The FOV varied fairly linearly with luminance. An empirical formula for this relationship is:

$$
\theta_{\mathrm{FOV}}=34^{\circ}-0.0013 \mathrm{~L}
$$

where $\theta_{\mathrm{Fov}}$ is the upper FOV angle in degrees and $\mathrm{L}$ is the luminance in $\mathrm{cd} / \mathrm{m}^{2}$. The dashed lines represent one standard deviation above and below the mean, encompassing $77 \%$ of the data. Many factors may cause such a variation in the level of photosensitivity of different people. These factors may include nationality, eye color, skin type, and facial bone structure. A classification by eye color shows there is quite a bit of overlap between the darker and lighter colored groupings. Although the subjects having darker irides had slightly more lid opening in general, there were subjects with blue eyes who had the largest opening, and this appeared to be more a function of pupil size. The permanent structure of the face causes some people to have a natural limit upon their vertical visual field. Those with "deep set" eyes tend to have a smaller acceptance angle regardless of the brightness, presumably due to blockage by the brow ridge and cheeks. The lower extreme of data is mostly comprised of people with deep set eyes.

\section{Sunglasses}

In our studies using UV detectors mounted in the eyes of a mannequin, and in similar studies of Rosenthal ${ }^{40}$, we found that even with UV blocking lenses, the UVR reaching the "cornea" (the detector) was about $18-25 \%$ as a result of energy arriving from above the frame or from the sides. Since dark lenses increase lid opening and pupil opening, the UVR dose to the crystalline lens can be further increased. Therefore, the use of a simple assumption that sunglasses greatly filter UV-B may be erroneous.

\section{CONCLUSIONS}

Several factors determine UVR exposure of ocular structury 
and the most important are geometrical factors determined by lid opening and wavelength. A thorough understanding of these environmental and physiological factors are key to any risk assessment of UVR exposure of the human eye and for improved epidemiologic studies. Without a consideration of these factors, subjects could well be assigned to a "greater" rather than "lessor" cohort of exposed individuals

Outdoor sunlight exposure over a lifetime generally dwarfs any UVR exposure experienced in an indoor industrial occupational setting, thus it has been particularly important to accurately determine the ocular exposure experienced in the outdoor environment. Although, this determination might appear at first to be quite straightforward, it is unfortunately very complex due to the geometry of exposure and the changing UV spectrum and directions of sunlight. What may at first appear to be obvious with regard to ocular exposure, frequently turns out to be the opposite! The geometrical factors which affect ocular exposure play a major role in determining the nature of any age-related ocular changes where sunlight plays an etiological role. The locations of exposure across the comea and lens are determined by the palpebral fissure (the lid opening) and horizon sky UVR; and the retinal zones exposed depend upon the lid positions as well. The change in ambient UVR is greatly dependent upon the presence of clouds and the sun's elevation angle which varies not only each hour, but by season. The presence of clouds and haze redistribute the UVR in skylight and can actually increase the UV radiance ("brightness") of the horizon sky (and thereby increase ocular exposure) ${ }^{13,28}$. Although the ambient global measurement of UVR increases with higher altitudes, the horizon UVR within the ocular field-of-view actually decreases. Sky scattering at sea level is very substantial, and although the total UVR is less compared to a high mountain, the horizon-sky UVR radiance increases.

\section{REFERENCES}

1. United Nations Environment Program, World Health Organization, International Commission on Radiation Protection, Environmental Health Criteria No. 160, Ultraviolet Radiation, joint publication. World Health Organization, Geneva, 1994.

2. Young, R. W. Age Related Cataract, New York: Oxford University Press, 1991.

3. Jose JG \& Pitts DG (1985) Wavelength dependency of cataracts in albino mice following chronic exposure. Exp Eye Res, 41:545-563.

4. Pitts D G, Cullen A P, Hacker P D 1977 Ocular effects of ultraviolet radiation from 295 to $365 \mathrm{~nm}$ Invest Ophthalmol Vis Sci 16(10):932-939

5. Bachem A. (1956) Ophthalmic ultraviolet action spectra, Am J Ophthalmol, 41: 969-975.
6. Taylor HR, West SK, Rosenthal FS, Munoz B, Newland HS, Abbey H, \& Emmett EA (1988) Effect of ultraviolet radiation on cataract formation. New Engl J Med, 319:1429-1433.

7. Sliney, D.H., "Physical Factors in Cataractogenesis: Ambient Ultraviolet Radiation and Temperature," Invest Ophthalmol Vis Sci 1986; 27:781-790.

8. Sliney, D.H., Epidemiological studies of sunlight and cataract: the critical factor of ultraviolet exposure geometry, Ophthalmic Epidemiology, 1(2):107-119, 1994.

9. Weale, R. "Senile cataract, the case against light." Ophthalmology 90:420- 423, 1983; see also: Brit J Ophthalmol 66(1):31-34, 1982.

10. Harding, J.J. "The untenability of the sunlight hypothesis of cataractogenesis," Doc. Ophthalmologica, 88:345349, 1995.

11. Klein, B.E.K., Klein, R., and Linton, K.L.P. Prevalence of age-related lens opacities in a population, the Beaver Dam eye study. Ophthalmology, 99(4):546-552, 1992.

12. World Health Organization, The Effects of Solar UV Radiation on the Eye, Report of an Informal Consultation, Geneva 30 August - September 1993," Publication WHO/PBL/EHG/94.1, Program for the Prevention of Blindness Program, World Health Organization, Geneva, 1995.

13. Sliney, D. H., UV radiation ocular exposure dosimetry, $J$ Photochem Photobiol B31:69-77, 1995.

14. Zuclich J A., 1989 Ultraviolet-induced photochemical damage in ocular tissues. Health Phys 56(5):671-682

15. Pitts D G, 1974 The human ultraviolet action spectrum Am J Optom Physiol Optics 51(12):946-960

16. Ringvold A. Damage of the cornea epithelium caused by ultraviolet radiation, Acta Ophthalmologica, 61:898-907, 1983.

17. Sliney, D. H., Eye Protective Techniques for Bright Light, Ophthalmology 1983; 90:937-944.

18. Hedblom EE. Snowscape eye protection, Arch Environ Health, 2:685-704, 1961.

19. Wulf HC: Effects of ultraviolet radiation from the sun on the Inuit population. In: Circumpolar Health 93 (ed. Petursdottir G, Sigurdsson SB, Karlsson MM, Axelsson J). Arctic Medical Research 53: 416-422, 1994

20. Dolin P.J., Ultraviolet radiation and cataract: A review of the epidemiological evidence, $\mathrm{Br}$. J. Ophthalmol, and reprinted in Optometry Today Nov/Dec 1997, 78, 478482, 1994

21. Ham W T, Mueller H A, Ruffolo J J, Guerry D III, Guerry R K 1982 Action spectrum for retinal injury from near ultraviolet radiation in the aphakic monkey $A m J$ Ophthalmol 93(3): 299-306

22. Mainster M A 1978 Spectral transmission of intraocular lenses and retinal damage from intense light sources $\mathrm{Am}$ 
J Ophthalmol 85:167-170

23. Sliney D H and Wolbarsht M L 1980 Safety with Lasers and Other Optical Sources (New York: Plenum Publishing Corp)

24. Dillon J., The photophysics and photobiology of the eye, J Photochem Photobiol, 10, 23-40, 1991.

25. Urbach F, and Gange R W (Eds.) 1986 The Biological Effects of UV-A Radiation (Westport: Praeger Publishers)

26. Coroneo, M.T., M_ller-Stolzenburg, N.W. Ho, A., Peripheral light focussing by the anterior eye and the ophthalmohelioses, Ophthalmic Surg 22:705-711, 1991.

27. Coroneo, M.T., Pterygium as an early indicator of ultraviolet insolation: an hypothesis, Brit J Ophthalmol, 77:734739, 1993.

28. Sliney DH, UV Radiation Ocular Exposure Dosimetry, Doc Ophthalmologica, 88:243-254 (1995)

29. Forbes P D, and Davies P D, 1982, Factors that Influence Photocarcinogenesis, in: (J.A. Parrish, M. L. Kripke, and W.L. Morison, Eds.) Chapter 7, Photoimmunology (New York: Plenum Publishing Corp)

30. DeGruijl FR, van der Leun JC: Estimate of the wavelength dependency of ultraviolet carcinogenesis in humans and its relevance to the risk assessment of stratospheric ozone depletion. Health Physics 67: 317-323, 1994.

31. Duchêne A S, Lakey J R A, and Repacholi M H, 1991 IRPA Guidelines on Protection against Non-lonizing Radiation (New York, McMillan)

32. Anderson DE, Skinner PE, Studies on bovine ocular squamous carcinoma ("cancer eye"). XI. Effects of sunlight. J Animal Sci 20:474-479, 1961.
33. Gelatt K.N. Veterinary Ophthalmology, Philadelphia, Lea and Febiger, 2nd Edn., pp. 626-635, 1991.

34. McKinlay A F, Diffey B L 1987 A reference action spectrum for ultraviolet induced erythema in human skin. In: Passchier WF, Bosnjakovic BFM (Eds.), Human Exposure to Ultraviolet Radiation: Risks and Regulations, pp. 83-87 (New York: Excerpta Medica Division, Elsevier Science Publishers)

35. Parrish J A, Jaenicke K F, Anderson R R 1982 Erythema and melanogenesis action spectra of normal human skin Photochem Photobiol 36(2):187-191

36. Sliney D H, 1991 Measurement of light and the geometry of exposure of the human eye. In: Marshall $\mathrm{J}(\mathrm{ed})$ The Susceptible Visual Apparatus, volume 16 in series on Visual and Visual Dysfunction, pp 23-39, (New York: McMillan Press)

37. Ham W T Jr 1983 The photopathology and nature of the blue-light and near-UV retinal lesion produced by lasers and other optical sources in M L Wolbarsht ed. Laser Applications in Medicine and Biology (New York: Plenum Publishing Corp)

38. Deaver, D.M., Davis, J., Sliney, D.H., Vertical visual fields-of-view in outdoor daylight, Lasers and Light, Vol 7(2/3):121-125 (1996).

39. International Commission on Non-Ionizing Radiation Protection (ICNIRP) 1997 Guidelines on limits of exposure to optical radiation from 0.38 to $3.0 \mathrm{~m}$, Health Physics.

40. Rosenthal, F.S., Bakalian A.E., and Taylor, H.R., The effect of prescription eyewear on ocular exposure to ultraviolet radiation, Amer J Publ Health, 78:72-74, 1988.

Table 1. Reflectance of ACGIH-Effective Solar UV-B from Terrain Surfaces [14,17].

\begin{tabular}{lr}
\hline Representative Terrain Surfaces & $\begin{array}{c}\text { Diffuse Reflectance } \\
\text { ACGIH-Weighted Solar UV-B }\end{array}$ \\
\hline Green Mountain Grassland & $0.8-1.6 \%$ \\
Dry, parched Grassland & $2-3.7 \%$ \\
Wooden Boat Dock & $6.4 \%$ \\
Black Asphalt & $5-9 \%$ \\
Concrete Pavement & $8-12 \%$ \\
Atlantic Beach Sand (Dry) & $15-18 \%$ \\
Atlantic Beach Sand (Wet) & $7 \%$ \\
Sea Foam (Surf) & $25-30 \%$ \\
Aged, "Dirty" Snow & $50 \%$ \\
Fresh Snow & $88 \%$ \\
\hline
\end{tabular}




\section{ANNEX I CIE PHOTOBIOLOGICAL SPECTRAL BANDS AND
DOSIMETRIC CONCEPTS}

When considering photobiological effects, it is useful to employ the convention of the International Commission on Illumination (CIE) for spectral bands. The CIE has designated 315 to $400 \mathrm{~nm}$ as UV-A, 280 to $315 \mathrm{~nm}$ as UV-B, and 100-280 nm as UV-C (CIE, 1970). Light (visible radiation) overlaps the UV-A and IR-A extending from $380 \mathrm{~nm}$ to at least $780 \mathrm{~nm}^{1,2)}$. [IR-A extends from $770 \mathrm{~nm}$ to 1400 $\mathrm{nm}$; IR-B, from 1400 to $3000 \mathrm{~nm}$; and IR-C from $3000 \mathrm{~nm}$ to $1 \mathrm{~mm}$.]

From a biological point-of-view, wavelengths below $180 \mathrm{~nm}$ (vacuum UV) are of little practical significance since they are readily absorbed in air. UV-C wavelengths are more photochemically active, because these wavelengths correspond to the most energetic photons, are strongly absorbed in certain amino acids and therefore by most proteins; whereas, UV-B wavelengths are somewhat less photochemically active, but more penetrating in most tissues. UV-A wavelengths are far less photobiologically active, but are still more penetrating than UV-B wavelengths and often play an interactive role when exposure occurs following UV-B exposure.

Although useful, it is important to keep in mind that these photobiological spectral bands are merely a "short-hand" notation, and they can be used to make general (but not absolute) statements about the relative spectral effectiveness of different parts of the UV spectrum in producing effects. The dividing lines, while not arbitrary, are certainly not fine dividing lines between wavelengths which may or may not elicit a given biological effect. One should always provide a wavelength band or spectral emission curve for the UV source being used and not rely totally on these spectral terms. There are also many authors who use $320 \mathrm{~nm}$ rather than the CIE defined dividing line of $315 \mathrm{~nm}$ to divide UV-A from UV-B. Some authors also may divide the UV-A band into two regions: UV-A1 and UV-A2, with a division made at about $340 \mathrm{~nm}$. For this reason, the exposure limits often overlap bands or do not even make use of them.

\section{Dosimetric Concepts}

Unlike most ionizing radiations and radiofrequency (RF) radiation, UVR--like most optical radiation--is absorbed very superficially and penetration depth in the skin or cornea is generally less than $1 \mathrm{~mm}$ and for UV-C only a few cell layers. For this reasons a surface dose concept rather than an a volumic dose is conventionally applied in the photobiological literature. The product of the surface exposure dose-rate and the exposure duration always must result in the same exposure dose (in joules-per-unit area at the tissue of interest) to produce a threshold injury. This is termed the Rule of Reciprocity, or the Bunsen-Roscoe Law). Repair mechanisms, recombination over long periods (normally hours) will lead to reciprocity failure. By contrast, thermal injury is a rate process, dependent upon the volumic absorption of energy across the spectrum, and therefore does not follow the reciprocity rule. Thermal effects show very broad spectral dependence and a spot-size dependence. Hence, the thresholds for biological injury and human exposure limits for purely photochemical injury are expressed as a surface exposure dose, known as radiant exposure. The product of the exposure dose rate, the irradiance $\mathrm{E}$ in $\mathrm{W} / \mathrm{m}^{2}$ or $\mathrm{W} / \mathrm{cm}^{2}$, and the exposure duration $\mathrm{t}$ is the radiant exposure $\mathrm{H}$ expressed in $\mathrm{J} / \mathrm{m}^{2}$ or $\mathrm{J} / \mathrm{cm}^{2}$, i.e.,

$$
\mathbf{H}=\mathrm{Et}
$$

Luminous exposure (photometric) and radiant exposure $\mathrm{H}$ are both quantities used to describe a total exposure dose from a flashlamp or from a more lengthy exposure. While both $\mathrm{E}$ and $\mathrm{H}$ may be defined over the entire optical spectrum, the luminous exposure is only defined over the visible spectrum and is therefore not of value in UV photobiology. Where radiant energy is more penetrating, as in the visible and IR-A spectral bands, it is sometimes useful to apply the radiometric concepts of fluence and fluence rate which includes not only incident radiant energy passing through a reference area, but the backscatter as well. For all photobiology, it is necessary to employ an action spectrum for photochemical effects.

As with any photochemical injury mechanism, one must consider the action spectrum, which describes the relative effectiveness of different wavelengths in causing a photobiological effect. For example, the UV safety function $S(\lambda)$ is also an action spectrum which is an envelope curve for protection of both eye and skin.

The $S(\lambda)$ curve is an action spectra which is used to spectrally weight the incident UVR to determine an effective irradiance for comparison with the threshold value or exposure limit. With modern computer spread-sheet programs, one can readily develop a method for spectrally weighting a lamp's spectrum by a variety of photochemical action spectra. The computation may be tedious, but straightforward:

$$
\mathrm{E}_{\mathrm{cff}}=\mathrm{ES}(\lambda)
$$

The EL is then expressed as a permissible effective irradiance $\mathrm{E}_{\text {eff }}$ or an effective radiant exposure. One then can compare different sources to determine relative effectiveness of the same irradiance from several lamps for a given action spectrum.

\section{Radiometric Quantities}

The following radiometric quantities may be used in photo- 
dermatology, and are briefly summarized here:

a Irradiance (surface dose rate) and radiant exposure (surface dose) are units specifying power or energy incident upon a plane. These quantities are the dose rate (irradiance) and exposure dose (radiant exposure) that are the most fundamental dose quantities used in all of photobiology. The units most commonly used are $\mathrm{W} / \mathrm{cm}^{2}$ and $\mathrm{J} / \mathrm{cm}^{2}$, respectively. $1 \mathrm{~W}=1 \mathrm{~J} / \mathrm{s}$.

b Fluence rate and fluence are used in some very sophisticated studies, where the internal surface dose with backscatter is included. These quantities are used correctly most often in theoretical studies of dose distribution and where photochemistry at the molecular level in tissue is enhanced as a result of multiple scattering events in tissue. Unfortunately, these terms are frequently misused to mean irradiance and radiant exposure because the units of $W / \mathrm{cm}^{2}$ and $\mathrm{J} / \mathrm{cm}^{2}$ are the same.

c Radiance (irradiance per solid angle) is an important quantity used by physicists in specifying a source. This quantity limits the ability of lenses and reflective optics in concentrating a light source. For, example, a xenon-arc lamp has a very high radiance and its energy can be focussed to produce a very high irradiance on a target tissue. By contrast, a fluorescent lamp tube has a much lower radiance, and its energy cannot be focussed to a high concentration. The units are $W /\left(\mathrm{cm}^{2} \mathrm{sr}\right)$.

$\mathrm{d}$ Radiant Intensity (power per solid angle) is used to indi- cate how collimated a light source really is. Although useful for specifying searchlights, it normally has very limited use in photobiology. The units are $\mathrm{W} / \mathrm{sr}$.

e Spectral quantities (units per wavelength) are used for specifying the energy, power or irradiance per wavelength interval. When calculating a photobiologically effective dose the spectral quantity must be multiplied by the action spectrum. Examples: spectral radiant power, spectral irradiance, spectral radiant exposure, etc. The units for each quantity are modified by adding "per nanometer," e.g., $\mathrm{W} / \mathrm{cm}^{2}$ becomes $\mathrm{W} /\left(\mathrm{cm}^{2} \mathrm{~nm}\right)$.

f Photon (Quantum) quantities (units of photons) are used primarily in theoretical studies, and in photochemistry. In this case the radiant exposure is specified in photons $/ \mathrm{cm}^{2}$ and irradiance is specified in photon/( $\left.\mathrm{cm}^{2} \mathrm{~s}\right)$.

\section{REFERENCES}

1. Commission International de l'Eclairage (International Commission on Illumination). International Lighting Vocabulary, 4th ed, 1987; Pub. CIE No. 17: E-1.1. CIE, Vienna.

2. Commission International de l'Eclairage (International Commission on Illumination). Standardization of the term UVA-1, UVA-2 and UVB, 1999; Pub. CIE No. 124-1999, CIE, Vienna. 